\title{
artigo
}

Caldas, G.R.F.; Alencar, A.P.A.; Lopes da Silva, C.R.; Oliveira, M.S.S.; Grangeiro Silva, E.M.; Lira, P.F.;

Puericultura na atenção primária a saúde: problemas evidenciados pelos enfermeiros

\section{Puericultura na atenção primária a saúde: problemas evidenciados pelos enfermeiros}

\author{
Childcare in primary health care: problems evidenced by nurses \\ El cuidado infantil en la atención primaria de salud: problemas que evidencian por enfermeiras
}

\begin{abstract}
RESUMO
Objetivou-se evidenciar os principais problemas identificados pelo enfermeiro durante a consulta de puericultura na atenção primária a saúde. Trata-se de uma revisão sistemática da literatura com metassíntese, com utilização do protocolo PRISMA para identificação da busca e seleção dos documentos. Realizou-se buscas na SciELO e LILACS, apresentando 61 documentos. Após critérios de inclusão: artigos disponíveis gratuitamente, publicados entre 2015 a 2020, em língua portuguesa, inglesa ou espanhola e exclusão: não apresentassem a tematica central da pesquisa, repetitivos, dissertações, teses e resumos, resultando em um total de 10 artigos. Os problemas identificados durante a puericultura seguiam a mesma linha, destacando-se problemas respiratórios, nutricionais, gastrintestinais e dermatológicos. Foi possível analisar a importância da realização da puericultura pelo profissional de enfermagem, utilizando de práticas de vigilância, atenção, escuta qualificada, criação de vínculo, estabelecimento de confiança, percepção de vulnerabilidades e acompanhamento constante, para que então, possa identificar necessidades e problemas que acometem as crianças.
\end{abstract}

DESCRITORES: Enfermagem; Puericultura; Atenção Primária à Saúde.

\section{ABSTRACT}

The objective was to highlight the main problems identified by nurses during childcare consultation in primary health care. It is a systematic review of the literature with meta-synthesis, using the PRISMA protocol to identify the search and selection of documents. SciELO and LILACS searches were carried out, presenting 61 documents. After inclusion criteria: articles available for free, published between 2015 and 2020, in Portuguese, English or Spanish and exclusion: did not present the central theme of the research, repetitive, dissertations, theses and abstracts, resulting in a total of 10 articles. The problems identified during childcare followed the same line, highlighting respiratory, nutritional, gastrointestinal and dermatological problems. It was possible to analyze the importance of carrying out childcare by the nursing professional, using practices of surveillance, attention, qualified listening, bonding, establishing trust, perception of vulnerabilities and constant monitoring, so that, then, you can identify needs and problems that affect children.

DESCRIPTORS: Nursing; Childcare; Primary Health Care.

\section{RESUMEN}

El objetivo fue destacar los principales problemas identificados por las enfermeras durante la consulta de puericultura en la atención primaria de salud. Es una revisión sistemática de la literatura con meta-síntesis, utilizando el protocolo PRISMA para identificar la búsqueda y selección de documentos. Se realizaron búsquedas SciELO y LILACS, presentando 61 documentos. Después de los criterios de inclusión: artículos disponibles de forma gratuita, publicados entre 2015 y 2020, en portugués, inglés o español y exclusión: no presentó el tema central de la investigación, repetitivo, disertaciones, tesis y resúmenes, resultando un total de 10 artículos. Los problemas identificados durante el cuidado infantil siguieron la misma línea, destacando problemas respiratorios, nutricionales, gastrointestinales y dermatológicos. Se pudo analizar la importancia de llevar a cabo el cuidado del niño por parte del profesional de enfermería, utilizando prácticas de vigilancia, atención, escucha calificada, vinculación, establecimiento de confianza, percepción de vulnerabilidades y seguimiento constante, para que, luego, se puedan identificar necesidades y problemas que afectar a los niños.

DESCRIPTORES: Enfermería; Cuidado de niños; Primeros auxilios.

RECEBIDO EM: 31/10/2020 APROVADO EM: 18/11/2020 


\section{Geovanna Renaissa Ferreira Caldas}

Acadêmica de Enfermagem do Centro Universitário de Juazeiro do Norte (UNIJUAZEIRO).

ORCID: 0000-0001-9820-309X

\section{Ana Paula Agostinho Alencar}

Enfermeira. Mestre em Ciências da Saúde, Especialista em Gestão na Atenção à Saúde e Qualidade no cuidado e segurança do paciente, Pesquisadora do grupo clínica, cuidado e gestão em saúde GPCLIN da Universidade Regional do Cariri (URCA), Coordenadora de estágio do Curso Vigilância em Saúde da escola Técnica do SUS-ETSUS, Enfermeira assistencial.

ORCID: 0000-0002-6262-4279

\section{Cicero Rafael Lopes da Silva}

Enfermeiro. Especialista em Enfermagem Dermatológica, Pós graduando em Docência do Ensino Superior - UNIJUAZEIRO e em Gerontologia e saúde do idoso - FAVENI, Docente da graduação em Enfermagem - UNIJUAZEIRO.

ORCID: 0000-0001-8819-5380

\section{Maria do Socorro Santos de Oliveira}

Enfermeira. Especialista em Enfermagem Obstétrica, Docente de Enfermagem - UNIJUAZEIRO.

ORCID: 0000-0001-9392-2378

\section{Édylla Monteiro Grangeiro Silva}

Enfermeira. Especialista em Saúde Coletiva com ênfase na Estratégia Saúde da Família, Preceptora do Curso de Enfermagem da UNIJUAZEIRO.

ORCID: 0000-0003-2630-8997

\section{Petrúcya Frazão Lira}

Enfermeira. Mestre em Ciências da Educação, Especialista em Gestão de Redes, Docente da UNIJUAZEIRO, Coordenadora da Escola Técnica do SUS Dr. Antonio Marchet Callou.

ORCID: 0000-0001-9539-066X

\section{INTRODUÇÃO}

$\mathbf{E}$ m consequências dos elevados números de mortalidade infantil, as Nações Unidas instituíram como objetivo, a redução dessa mortalidade de 2/3 até o ano de 2015. Em 2010, o Ministério da Saúde (MS) implementou a vigilância da mortalidade, para que as equipes de saúde identifiquem as necessidades apresentadas pelas crianças vinculadas a sua área, podendo realizar intervenções com enfoque nos problemas encontrados, para com isso, reduzir o número de mortes infantis ${ }^{1}$.

A Organização Mundial de Saúde (OMS) define e exemplifica que a taxa de mortalidade infantil permite avaliar os cuidados de saúde realizados com o público infantil, a eficácia das práticas de assistência, a influência de todo o convívio social e cultural e da situação econômica, levando em consideração as possíveis variáveis, os prováveis e futuros problemas de saúde e as necessidades apresentadas
A puericultura é uma prática que pode ser utilizada pelo enfermeiro

para acompanhar

o crescimento e

desenvolvimento

físico, motor e

cognitivo da criança ou expostas por essa população especifica, considerando suas peculiaridades e auxiliando na criação e definição de políticas públicas voltadas para a área materna e infantil ${ }^{2}$.

A Política Nacional de Atenção Integral à Saúde da Criança (PNAISC), considerando cuidado integralizado e humanizada ao público infantil na Atenção Primária à Saúde (APS), estruturando-se em 07 eixos, a atenção humanizada ao parto e recém-nascido com capacitação dos profissionais, aleitamento materno e complementar, acompanhamento do crescimento e desenvolvimento infantil, atenção a doenças e agravos por meio de diagnóstico precoce, manejo e prevenção, atenção a situações de violências e acidentes, atenção a vulnerabilidades e deficiências e a prevenção do óbito fetal e materno ${ }^{3}$.

A puericultura é uma prática que pode ser utilizada pelo enfermeiro para acompanhar o crescimento e desenvolvimento físico, motor e cognitivo da criança, através 
de cuidados como, avaliação dos reflexos, avaliação dos marcos do desenvolvimento infantil, cobertura vacinal completa, orientações sobre amamentação exclusiva, amamentação complementada, introdução de novos alimentos, avaliar perímetros cefálico, torácico e estatura, e a prevenção de doenças e problemas de saúde que são frequentes nas crianças. Inclui-se ainda, o acompanhamento familiar, considerando as vulnerabilidades econômicas, sociais e de saúde física e mental da família, especialmente da mãe, analisando as condutas que podem ser realizadas, por meio de aconselhamento e educação em saúde, para proporcionar uma boa qualidade de vida para a criança ${ }^{4}$.

O Ministério da Saúde recomenda a realização de, no mínimo, sete consultas de puericultura no primeiro ano de vida, esse apresentando-se, como um cuidado indispensável para a equipe durante a realização da assistência, em busca de garantir um cuidado integral, humanizado, universal, individual e/ou coletivo, garantindo a equidade e atuando sob as diretrizes e princípios doutrinários do Sistema Único de Saúde (SUS) ${ }^{5-6}$.

Surgiu a seguinte questão norteadora: Quais os principais problemas de saúde identificados pelos enfermeiros durante a consulta de puericultura?

Destaca-se a relevância por abordar importantes informações sobre a saúde infantil, para elaborações futuras estratégias, em busca da melhoria na qualidade da assistência. Em virtude disso, o objetivo do estudo é evidenciar os principais problemas identificados pelo enfermeiro durante a consulta de puericultura na atenção primária a saúde.

\section{MÉTODO}

O método pode ser definido como um processo sistematizado, onde são adotadas várias etapas determinando todo um caminho a ser seguido, com o intuito de conseguir obter um resultado e atingir os objetivos propostos, além ainda de atingir o conhecimento 7 .

O presente estudo, trata-se de uma revi-
O Ministério da

Saúde recomenda

a realização de, no

mínimo, sete consultas

de puericultura no

primeiro ano de vida,

esse apresentando-se,

como um cuidado

indispensável para

a equipe durante

a realização da

assistência, em busca

de garantir um

cuidado integral,

humanizado,

universal, individual

e/ou coletivo,

garantindo a equidade

e atuando sob as

diretrizes e princípios

doutrinários do

Sistema Único de

Saúde (SUS) são sistemática da literatura com metassíntese. Esse tipo de pesquisa segue protocolos que busca proporcionar um conhecimento lógico e cientifico, através de objetivos estabelecidos pelos autores e o uso da metodologia para a obtenção de resultados com altos níveis de evidências, por intermeio da metassíntese, utilizada para associar estudos qualitativos, localizando e dividindo-se em temas, áreas ou tópicos ${ }^{8}$.

Uma abordagem qualitativa de um estudo, segue o raciocínio de investigar, avaliar e interpretar os dados buscados, expondo os resultados juntamente om detalhes sobre o que foi investigado?.

Realizou-se a pesquisa referida na plataforma onde estão indexadas várias bases de dados: Biblioteca Virtual em Saúde (BVS), citando como bases: Scientific Electronic Library Online (SciELO), Biblioteca Virtual en Salud Enfermería (BDENF-Enfermagem) e Literatura Latino-americana e do Caribe em Ciências da Saúde (LILACS), por intermeio da utilização dos descritores em ciências da saúde (DeCS): "puericultura", "atenção primária a saúde" e "enfermagem", em associação do operador booleano AND.

Dentre os critérios de inclusão, cita-se os estudos: $1^{\circ}$ ) disponíveis integralmente de forma gratuita, $2^{\circ}$ ) publicados entre 2015 a junho de $2020,3^{\circ}$ ) publicados em português, inglês ou espanhol. Os critérios de exclusão definiram-se em estudos que não apresentassem a tematica central, que não respondessem a questão norteadora, repetitivos, dissertações, teses, documentários, monografias, projetos, resumos simples ou expandidos.

Após pesquisa e aplicação dos filtros de ano e linguagem, realizou-se leitura dos resumos de todos os estudos restantes, para melhor selecionar os artigos que se enquadravam nos critérios de inclusão e exclusão elegidos, para constituir o seguinte estudo.

No que diz respeito a parte de aspectos éticos da pesquisa para as revisões, é dispensável a submissão ao Comitê de ética e pesquisa com seres humanos. Pois, os estudos utilizados para a escrita da revisão já passaram pela a apreciação e apresentam-se disponíveis online nas bases de dados. 
Ao realizar a busca na BVS, foi filtrada apenas a base de dados LILACS e

\section{Tabela 01 - Fluxograma de escolha dos documentos.}

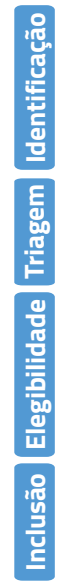

Ao realizar pesquisa dos DeCS na base de dados LILACS $(n=48)$

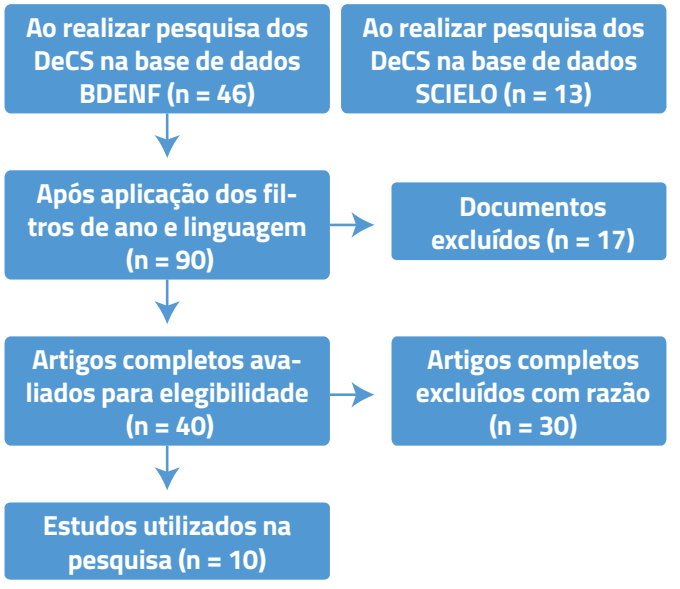

Fonte: Protocolo PRISMA
BDENF-Enfermagem, obtendo uma totalidade de 94 documentos. Ao efetivar a mesma busca na base de dados SciELO, foram obtidos 13 documentos, resultando em 117 artigos. Após a aplicação dos critérios de inclusão e exclusão previamente estabelecidos, restaram 40 documentos, dos quais, apenas 10 abordavam a tematica central da pesquisa e fizeram parte da construção do estudo, dos quais, LILACS (08), BDEFN-Enfermagem (01) e SciELO (01).

Para demonstração de todo processo de busca e escolha dos documentos utilizados, utilizou-se um fluxograma baseado no protocolo PRISMA, ilustrado na tabela 01.

Os 10 documentos selecionados foram arranjados na tabela 02 , quanto ao autor e ano de publicação, objetivo e principais achados, organizados do para achado mais recente para o menos recente.

\section{Tabela 02 - Distribuídos por autor e ano, base de dados, título, tipo de estudo e periódicos}

\begin{tabular}{|c|c|c|c|c|c|}
\hline$N^{0}$ & Autores e ano & Base & Titulo & Tipo de Estudo & Periódicos \\
\hline 01 & $\begin{array}{l}\text { Hanzen IP, Zanotelli } \\
\text { SS, Zanatta EA, } 2019\end{array}$ & BDENF & $\begin{array}{l}\text { Diagnostics, interventions and Nursing } \\
\text { results to subsidy the Nursing consultation } \\
\text { of the child }\end{array}$ & Pesquisa-ação & Enfermagem em foco \\
\hline 02 & $\begin{array}{l}\text { Ferreira FA, Freitas } \\
\text { RSC, Santos MCS, } \\
\text { Silva SRM, Silva AM, } \\
\text { Santos MKS, } 2019\end{array}$ & LILACS & $\begin{array}{l}\text { Consulta de puericultura: problemas encon- } \\
\text { trados em menores de } 2 \text { anos }\end{array}$ & Retrospectivo & $\begin{array}{l}\text { Revista de Enferma- } \\
\text { gem UFPE online }\end{array}$ \\
\hline 03 & Vieira DS, et al, 2019 & SCIELO & $\begin{array}{l}\text { A prática do enfermeiro na consulta de pue- } \\
\text { ricultura na estratégia saúde da família }\end{array}$ & Observacional & Texto-Contexto \\
\hline 04 & $\begin{array}{c}\text { Dantas SLC, Rodri- } \\
\text { gues DP, Fialho AVM, } \\
\text { Barbosa EMG, Perei- } \\
\text { ra AMM, Mesquita } \\
\text { NS, } 2018\end{array}$ & LILACS & $\begin{array}{l}\text { Representações sociais de enfermeiros da } \\
\text { atenção primária à saúde sobre cuidado de } \\
\text { enfermagem no pós-parto }\end{array}$ & Pesquisa de campo & Cogitare Enfermagem \\
\hline 05 & $\begin{array}{l}\text { Brito GV, Albuquer- } \\
\text { que IM'AN, Ribeiro } \\
\text { MA, Ponte ECS, Mo- } \\
\text { reira RMM, Linhares } \\
\text { M das GC, } 2018\end{array}$ & LILACS & $\begin{array}{l}\text { Consulta de puericultura na estratégia saúde } \\
\text { da família: percepção de enfermeiros }\end{array}$ & Estudo exploratório & Revista APS \\
\hline 06 & $\begin{array}{l}\text { Yakuwa MS, Neill S, } \\
\text { de Mello DF, } 2018\end{array}$ & LILACS & $\begin{array}{l}\text { Estratégias de enfermeiros para a vigilância } \\
\text { à saúde da criança }\end{array}$ & Pesquisa de campo & Rev. Latino-Americana \\
\hline 07 & $\begin{array}{l}\text { Santos JS, Yakuwa } \\
\text { MS, Andrade RD, } \\
\text { Henrique NCP, de } \\
\text { Mello DF, } 2016\end{array}$ & LILACS & $\begin{array}{l}\text { Cuidado cotidiano da criança: necessidades } \\
\text { e vulnerabilidades na perspectiva de mães } \\
\text { adolescentes }\end{array}$ & Estudo exploratório & Rev. Eletr. Enferm. \\
\hline 08 & $\begin{array}{l}\text { Pereira M de M, et al, } \\
2015\end{array}$ & LILACS & $\begin{array}{l}\text { Nursing educational practice in primary heal- } \\
\text { th care aimed to healthy child development }\end{array}$ & Exploratório-desc. & Cogitare Enfermagem \\
\hline
\end{tabular}




\section{artigo}

Caldas, G.R.F.; Alencar, A.P.A.; Lopes da Silva, C.R.; Oliveira, M.S.S.; Grangeiro Silva, E.M.; Lira, P.F.;

Puericultura na atenção primária a saúde: problemas evidenciados pelos enfermeiros

Moura MAP, Rocha
SS da, Pinho DLM, LILACS
Guilhem D, 2015
Aires LC dos P, dos
Santos EKA, Costa R, LILACS
Borck M, Custódio ZA
de O, 2015
Fonte: Elaboração própria.
E posteriormente arranjados na tabela
03, apresentando os resultados encontra-
dos dentre os artigos analisados.
DISCUSSÃo
A importância da realização da pue-
ricultura

Facilidades e dificuldades dos enfermeiros

no cuidar da alimentação infantil na atenção básica

Seguimento do bebê na atenção básica: interface com a terceira etapa do método canguru
Pesquisa de campo O Mundo da Saúde

Exploratório-descritivo
Revista Gaúcha de Enfermagem
Ao acompanhar essas criança, é possível a realização de cuidados de prevenção, podendo ao enfermeiro então, atuar sobre as carências ou problemas encontrados 5 .

Vieira ${ }^{10}$ enfatiza o acompanhamento e vigilância da criança durante a realização da consulta de puericultura, considerando a importância da detecção em tempo oportuno das necessidades, por meio do acompanhamento do desenvolvimento infantil e problemas de saúde apresentados pelas crianças. A puericultura deve ser voltada para as necessidades, das quais, os cuidados devem ser sempre individualizados para cada criança e família ${ }^{11}$.

Considerando a nossa atual realidade,

\section{Tabela 03 - Resultados/Achados}

\section{Resultados}

Foram elencados um total de 19 Diagnósticos, citando-se: Alergia, Candidíase oral, Dermatites, Eritema, Amamentação prejudicada,

01 Crescimento e/ou Desenvolvimento prejudicado, Peso prejudicado, Cólica abdominal, Constipação, Diarreia, Vômito, Desenvolvimento infantil prejudicado, Febre, Infecções, Higiene prejudicada, entre outros.

Evidenciou-se 139 problemas, divididos em áreas, sendo, respiratória (tosse, resfriado, dispneia, asma e alergia respiratória), der-

02 matológica (dermatite, manchas, lesões, eritema, alergia, candidíase oral e inflamações) e gastrointestinais (diarreia, falta de apetite, constipação, cólicas, desnutrição, refluxo e fezes com coloração diferente).

Ao abordar o processo de vigilância do desenvolvimento infantil, realizada pelo enfermeiro durante a puericultura. Percebe-se que

03 essas ações permitem o acompanhamento da criança e a avaliação de reflexos presentes ou ausentes, baixo peso ou peso elevado, o perímetro cefálico e a atenção para a microcefalia, entre outros.

A pesquisa apresenta 3 eixos, sobressaindo-se $01^{\circ}$ eixo, cuidados de enfermagem atribuídos pelos enfermeiros para as mães e

04 crianças, na busca de prevenir ou atuar sobre os problemas recorrente na puericultura, por exemplo, o aleitamento materno exclusivo, a higiene de forma preservada, orientações gerais proporcionando educação em saúde, dentre outras.

Um dos pontos abordados é o saber e a experiência frente a puericultura, pois dentre os possíveis problemas, os enfermeiros referiram mal desenvolvimento físico ou cognitivo, baixo peso, doenças prevalentes da infância, crescimento prejudicado, etc.

Enfatizou-se ações estratégicas voltadas para avaliação e atenção de algumas queixas, utilizando da puericultura como um facilitador, observando se a familia possui condição para garantir uma estrutura e um ambiente que a criança possa crescer e desenvolver sem interferir futuramente na saúde daquela criança e a criação de vínculo entre mãe e filho.

Dentre as percepções analisadas, destaca-se as vulnerabilidades e dificuldades relatadas pelas adolescentes, como falta de ex-

07 periência com cuidado a criança, que gera dificuldades na identificação de possíveis problemas de saúde, otites e inflamações que causam dores e amamentação difícil, levando ao desmame precoce e o favorecimento de sobrepeso ou desnutrição.

Sobressai-se um conjunto de ações, a higienização e os riscos de mantê-la prejudicada, imunização e as doenças advindos do atraso

08 ou da não vacinação, a nutrição e as fragilidades que geram baixo peso ou peso elevado, avaliando e orientando possíveis razões para atrasos físicos ou mentais, através do uso da caderneta e dos parâmetros estabelecidos.

Evidencia problemas específicos entre as crianças, voltados para a área de alimentação e amamentação, apresentando rejeita da

09 amamentação, principalmente pelas mães que oferecem outros tipos de leite cedo, aumentando risco para alergias, problemas gastrointestinais e obesidade. Aborda ainda os aspectos econômicos e o quanto isso afeta na qualidade da alimentação.

Identifica-se carência de suporte familiar, higienização, sinais de maus tratos, vulnerabilidades biopsicossociais, relacionamento e

10 vínculo entre a família. Destaca-se, a importância do acompanhamento de bebês que comparecem a puericultura em estado de baixo peso, PIG e prematuro, que apresentam maiores necessidades e maiores riscos.

Fonte: Elaboração própria. 
o enfermeiro vem se tornando o principal desenvolvedor da prática, por consequência do acolhimento e do vínculo com os pacientes, através do emprego de uma escuta adequada, atentando ao que ele apresenta e considerando suas particularidades ${ }^{12}$. Durante a realização da consulta de enfermagem, deve ser seguido uma Anamnese detalhada, Exame físico bem estruturado, a verificação dos dados antropométricos e do estado nutricional, investigar se a imunização está atualizada, e se o Desenvolvimento Neuropsicomotor (DNPM) está sendo assegurado ${ }^{13}$.

Sabe-se que os primeiros anos de vida da criança são de extrema importância para as superfícies dos desenvolvimentos cognitivo, motor, socioafetivos e comportamentais. Por essa razão, é importante a avaliação e acompanhamento do desenvolvimento durante a infância, em busca de evitar possíveis atrasos e problemas no $\mathrm{DNPM}^{14}$.

\section{A consulta e os diagnósticos de en- fermagem}

A enfermagem costuma possuir contato frequente com seus pacientes, permitindo a criação de vínculo, facilitando a assistência, através do Processo de Enfermagem $(\mathrm{PE})^{15}$.

Para garantia de uma ação plena e completa, o Enfermeiro deve apresentar conhecimentos teóricos, prático e noção do contexto econômico família, social, cultural e religioso ${ }^{16}$. Santos ${ }^{17}$, apresenta em seu estudo a importância dessa noção da situação de vida que a família se encontra, apresentando dificuldades enfrentadas por mães adolescentes e a relevância do quanto essa situação afeta a vida da criança, podendo gerar problemas de saúde relacionados a falta de conhecimento ou de confiança da adolescente.

Hanzen, Zanotelli e Zanatta ${ }^{18}$ expuseram diagnósticos de enfermagem, elencando as alergias, candidíase oral, dermatites em geral, eritemas por diferentes causas, amamentação prejudicada, crescimento e/ou desenvolvimento prejudicado, peso prejudicado, cólicas, diarreia, constipação, vômito, higiene, tosse, congestão nasal e febre. Confirmando isso, $\operatorname{Costa}^{19}$ possui a mesma proposta de apresentar diagnósticos para crianças, avaliando a higiene, eliminações, amamentação, integridade da pele, crescimento e/ou peso. Entretanto, o estudo inclui outras opções, como o sono prejudicado, vínculo e processo familiar, regime de imunização, suplementação, entre outros.

\section{A enfermagem \\ costuma possuir contato frequente} com seus pacientes, permitindo a criação de vínculo, facilitando a assistência, através do Processo de Enfermagem (PE)

\section{Principais problemas de saúde evi- denciados nas crianças}

Ferreira ${ }^{13}$ exibe mais de 100 problemas de saúde, divididos entre as categorias de problemas respiratórios, dermatológicos, gastrintestinais, hematológicos e cardiológicos. Dentre os quais, a maioria estava incluído em resfriado comum, tosse produtiva, dermatite de contato e diarreia. Reforçando isso, um estudo ${ }^{20}$ relata que o maior índice está relacionado ao sistema respiratório, hipersensibilidade e sistema gastrointestinal.
Faz-se necessário um acompanhamento criterioso, atentando-se a sinais de carência, má higienização, maus tratos e vulnerabilidades ${ }^{21}$. Os maus tratos ocorrem na sua maioria dos casos, dentro do setor familiar, podendo incidir de forma física, emocional/mental, sexual, explorações, abandono ou negligência ${ }^{22}$.

As pesquisas de Dantas ${ }^{23}$, Brito ${ }^{24}$ e Yakuwa, Neill e Mello ${ }^{25}$ referem resultados semelhantes, como o desenvolvimento prejudicado, seja ele físico ou cognitivo, crescimento retardado, diarreia, vômitos e aleitamento materno prejudicado, podendo provocar desnutrição na criança, desidratação e baixo peso ou a troca da amamentação por uso de formulas que podem gerar ganho de peso exacerbado.

Além de todas essas problemáticas já citadas, Pereira ${ }^{26}$ aborda esses parâmetros, mas inclui ainda, a importância do uso da caderneta para o acompanhamento dessas crianças e as doenças relacionadas ao atraso da vacinação ou a prática de não vacinar.

Moura, Rocha, Pinho e Guilhem ${ }^{27}$ apontam os aspectos financeiros e como eles influenciam na alimentação que a criança recebe, onde muitas vezes, é dado preferência para outras prioridades da parte financeira.

\section{CONCLUSÃO}

Foi possível analisar a importância da realização da puericultura pelo profissional de enfermagem, utilizando de práticas de vigilância, atenção, escuta, vínculo, confiança, percepção de vulnerabilidades e acompanhamento constante.

As crianças apresentam, ou as família relatam, as mesmas queixas. Onde, problemas respiratórios, dermatológicos, gastrintestinais e nutricionais apresentam-se com uma maior prevalência. Percebe-se ainda, que certos problemas de saúde acarretam o aparecimento de novas queixas ou a piora do quadro clínico, por exemplo, crianças que fazem uso de outros leites, são mais propensas ao aparecimento de alergias e dermatites.

Corrobora-se a relevância da puericultura para a identificação de proble- 


\section{artigo}

Caldas, G.R.F.; Alencar, A.P.A.; Lopes da Silva, C.R.; Oliveira, M.S.S.; Grangeiro Silva, E.M.; Lira, P.F.i

Puericultura na atenção primária a saúde: problemas evidenciados pelos enfermeiros

mas e queixas frequentes do público infantil, carecendo de sensibilização e desempenho dos profissionais para as ações de prevenção, promoção, atuação frente as necessidades encontrados e orientaçôes aos familiares sobre tais queixas frequentes da infância, por meio da realização da educação em saúde e uma assistência de qualidade.

\section{REFERÊNCIAS}

1. Oliveira CM, Frias PG, Bonfim CV, Antonino VCS, Nascimento JDT, Medeiros ZM. Assessment of infant mortality surveillance: case study. Rev. Bras. Saúde Mater. Infant. Vol. 17, n. 04, pag. 801-815, Dec. 2017.

2. Kropiwiec MV, Franco SC, Amaral AR. Fatores associados à mortalidade infantil em município com índice de desenvolvimento humano elevado. Rev. paul. pediatr. Vol. 35, n. 04, pag. 391-398, Dec, 2017.

3. Brasil. Ministério da Saúde. Portaria $n^{\circ} 1.130$, de 5 de agosto de 2015. Institui a Política Nacional de Atenção Integral à Saúde da Criança (PNAISC) no âmbito do Sistema Único de Saúde (SUS)., 2015.

4. Vieira DS, Santos Nathanielly Cristina Carvalho de Brito, Nascimento João Agnaldo do Collet Neusa, Toso Beatriz Rosana Gonçalves de Oliveira, Reichert Altamira Pereira da Silva. A prática do enfermeiro na consulta de puericultura na estratégia saúde da familia. Texto contexto - enferm. Vol. 27, n. 04, 2018.

5. Soares DG, Pinheiro MCX, Queiroz DM, Soares DG. Implantação da puericultura e desafios do cuidado na estratégia saúde da família em um município do estado do ceará. Rev Bras Promoç Saúde, vol. 29, n. 01, pag. 132-138, Fortaleza, jan./mar., 2016.

6. Brasil. Ministério da Saúde. Secretária de Atenção à Saúde. Departamento de Atenção Básica. Saúde da Criança: Crescimento e Desenvolvimento. Brasília, p.43-57, 2012. (Cadernos de Atenção Básica, nº 33).

7. Prodanov CC, Freitas EC. Metodologia do trabalho científico: Métodos e Técnicas da Pesquisa e do Trabalho Acadêmico. Universidade Freevale. Novo Hamburgo, 2013.

8. Galvão MCB, Ricarte ILM. Revisão sistemática da literatura: Conceituação, produção e publicação. Logeion: Filosofia da Informação, vol. 06, n. 01, pag. 57-73, 15 de Set. 2019.

9. Lakatos EM, Marconi MA. Fundamentos de metodologia científica. Atlas, 5. Ed. São Paulo, 2003.

10. Vieira D de S, Dias TKC, Pedrosa RKB, Vaz EMC, Collet N, Reichert AP da S. Processo de trabalho de enfermeiros na vigilância do desenvolvimento infantil. REME - Rev Min. Enfermagem, vol. 23, 2019.

11. Benicio AL, Santana MDR, Bezerra IMP, Santos RR. Cuidado à criança menor de um ano: perspectiva da atuação do enfermeiro na puericultura. Rev enferm UFPE on line. Recife, vol. 10, n. 02, pag. 57684, fev., 2016.

12. Almeida ER, Moutinho CB, Carvalho SAS, Araújo MRN. Relato sobre a construção de um protocolo de enfermagem em puericultura na atenção primária. Rev enferm UFPE on line., Recife, 10( 2 ):683-91, fev., 2016.

13. Ferreira FA, Freitas RSC, Santos MCS, Silva SRM, Silva AM, Santos MKS. Consulta de puericultura: problemas encontrados em menores de 2 anos. Rev enferm UFPE on line, vol. 13, 2019.

14. Araujo LB, Quadros DA, Murata MPF, Israel VL. Avaliação neuropsicomotora de crianças de 0 a 5 anos em centros de educação infantil do ensino público. Rev. CEFAC, vol. 21, n. 03, 2019.

15. Krauzer IM, Adamy EK, Ascari RA, Ferraz L, Trindade L de L, Neiss M. Sistematização da assistência de enfermagem na atenção básica: o que dizem os enfermeiros?. Cienc. enferm. Vol; 21, n. 02, pag. 3138, 2015.

16. Silva SA, Fracolli LA. Avaliação da assistência à criança na Estratégia de Saúde da Familia. Revista Brasileira de Enfermagem, vol. 69, n. 01, pag. 54-61, feb. 2016.

17. Santos JS, Yakuwa MS, Andrade RD, Henrique NCP, de Mello DF. Cuidado cotidiano da criança: necessidades e vulnerabilidades na perspectiva de mães adolescentes. Revista Eletrônica de Enfermagem. Vol. 18, 2016.

18. Hanzen IP, Zanotelli SS, Zanatta EA. Diagnósticos, intervenções e resultados de enfermagem para subsidiar a consulta de enfermagem à criança. Enfermagem em Foco, artigo 01, pag. 16-21, 2019.

19. Costa $P$, et al. Diagnósticos de enfermagem em consultas de atenção primária à saúde de recém-nascidos. Revista Brasileira de Enfermagem, vol. 71, n. 06, pag. 2961-2968, Dezembro, 2018.

20. Rocha MJL, Caldeira AP. Morbidade referida para crianças assistidas por equipes de saúde da família na região nordeste de Minas Gerais, Brasil. Revista APS, vol. 19, n. 03, pag. 446-456, July/Sept. 2016.

21. Aires LC dos P, dos Santos EKA, Costa R, Borck M, Custódio ZA de O. Seguimento do bebê na atenção básica: interface com a terceira etapa do método canguru. Revista Gaúcha de Enfermagem, vol. 36, n. spe, Porto Alegre, 2015.

22. Calza TZ, Dell'Aglio DD, Sarriera JC. Direitos da criança e do adolescente e maus-tratos: epidemiologia e notificação. Revista SPAGESP, vol. 17, n. 01, pag. 14-27, Ribeirão Preto, 2016.

23. Dantas SL da C, Rodrigues DP, Fialho AV de M, Barbosa EMG, Pereira AMM, Mesquita NS de. Representações sociais de enfermeiros da atenção primária à saúde sobre cuidado de enfermagem no pós-parto. Cogitare enfermagem, vol. 23, n. 03, 2018.

24. de Brito GV, Albuquerque IM'AN, Ribeiro MA, Ponte ECS, Moreira RMM, Linhares $M$ das GC. Consulta de puericultura na estratégia saúde da familia: percepção de enfermeiros. Revista APS, vol. 21, n. 01, pag. 48-55, jan/mar, 2018.

25. Yakuwa MS, Neill S, de Mello DF. Estratégias de enfermagem para vigilância à saúde da criança. Revista Latino-Americana de Enfermagem, vol. 26, Julho, 2018.

26. Pereira $M$ de $M$, et al. Nursing educational practice in primary health care aimed to healthy child development. Cogitare Enferm., vol. 20, n. 04, pag. 761-767, Oct/dec, 2015.

27. Moura MAP, Rocha SS da, Pinho DLM, Guilhem D. Facilidades e dificuldades dos enfermeiros no cuidar da alimentação infantil na atenção básica. O Mundo da Saúde, vol. 39, n. 02, pag. 231-238, São Paulo, 2015 